\title{
Uma Ferramenta de Suporte ao Uso de Classificadores em Sistemas Embarcados
}

\author{
Lucas Tsutsui da Silva* Vinicius M. A. Souza* \\ Gustavo E. A. P. A. Batista** \\ * Instituto de Ciências Matemáticas e de Computação, Universidade de \\ São Paulo, São Carlos-SP, (e-mails: lucastsutsui@usp.br, \\ vmasouza@icmc.usp.br). \\ ** School of Computer Science and Engineering, University of New \\ South Wales, Sydney, Australia, (e-mail: gbatista@cse.unsw.edu.au)
}

\begin{abstract}
Computer systems are employed to solve existing problems in different contexts in society, such as health, industry, safety, transportation, and others. Many of these solutions are composed of sensors, embedded systems, and wireless communication. Moreover, researchers and practitioners have used machine learning models in embedded systems to perform complex tasks that can not be directly solved by traditional algorithms. In this work, we propose a software tool that converts classification models, trained with WEKA, to produce suitable classifiers to run in low-cost embedded systems with the option to use fixed-point representation. From an experimental evaluation with different embedded platforms, datasets and classifiers, we show consistent results of accuracy and a reduction of up to $92.9 \%$ in the classification time when using fixed-point representation.

Resumo: Sistemas computacionais são empregados para solucionar problemas existentes em diferentes contextos na sociedade, como saúde, indústria, segurança, transporte, entre outros. Muitas dessas soluções são compostas por sensores, sistemas embarcados e comunicação sem fio. Além disso, academia e indústria têm considerado a utilização de modelos de Aprendizado de Máquina em sistemas embarcados para realizar tarefas complexas que não podem ser diretamente solucionadas por algoritmos tradicionais. Neste trabalho, é proposta uma ferramenta de software que converte modelos de classificação, treinados com a ferramenta $W E K A$, para produzir classificadores que executarão em sistemas embarcados de baixo custo, com a opção de utilizar representação de ponto-fixo. A partir de uma avaliação experimental com diferentes plataformas embarcadas, conjuntos de dados e classificadores, são demonstrados resultados consistentes de acurácia e redução de até 92,9\% no tempo de classificação ao utilizar representação de ponto-fixo.
\end{abstract}

Keywords: Machine Learning; Classification; Embedded Systems; Embedded Classifiers.

Palavras-chaves: Aprendizado de Máquina; Classificação; Sistemas Embarcados;

Classificadores Embarcados.

\section{INTRODUÇÃO}

Tem sido cada vez mais popular o desenvolvimento de aplicações inspiradas na ideia de computação ubíqua, envolvendo emprego de sensores, sistemas embarcados e protocolos de comunicação sem fio para a automatização de diversas tarefas do cotidiano. Em paralelo, também houve um crescente interesse da comunidade científica e da indústria no desenvolvimento de aplicações práticas que utilizam conceitos relacionados a Inteligência Artificial, em especial Aprendizado de Máquina (AM), para tarefas que envolvem, por exemplo, a descoberta de conhecimento a partir de bases de dados. Nesse sentido, pesquisas indicam a possibilidade de uma frutífera convergência de ambos os temas para solução de tarefas mais complexas, a partir do desenvolvimento de aplicações com sensores e sistemas embarcados e que também empreguem modelos de AM.
Classificação automática é uma das principais tarefas provenientes de $\mathrm{AM}$, sendo utilizada em diferentes aplicações do mundo real. Muitas destas aplicações requerem que as decisões de classificação sejam tomadas em tempo real, isto é, com a chegada de novos dados deve-se satisfazer individualmente a restrição de tempo de cada solicitação de classificação (Stankovic, 1988). Desse modo, se faz necessário que classificadores sejam implementados diretamente em sistemas embarcados. Além disso, o processamento local é uma solução para sistemas que não possuem ou que evitam realizar comunicação com um sistema externo devido ao consumo de energia, por exemplo.

Na maior parte dos casos, os modelos de classificação são produzidos em outros sistemas computacionais e apenas o classificador gerado - de maneira off-board - é então implementado em um sistema embarcado. Isso é uma necessidade pois, em geral, a etapa de aprendizado de um preditor é mais complexa que a etapa de classificação. 
Além disso, treinar diferentes classificadores, para avaliálos e escolher o mais adequado, é uma tarefa que requer disponibilidade de memória e poder de processamento.

Considerando as restrições de memória e de processamento impostas por sistemas embarcados, é necessário que seja realizada uma análise criteriosa para a seleção de classificadores apropriados. A implementação desses classificadores pode exigir adaptações, principalmente em aplicações de baixo custo com restrições severas que afetam consideravelmente o desempenho do sistema.

Embora muitas aplicações exijam um tempo extremamente reduzido para processar os dados, são relatados na literatura trabalhos que empregam com sucesso sistemas embarcados para realizar tarefas de classificação em tempo real (Shi et al., 2009; Rúa et al., 2012; Tocchetto et al., 2014). Contudo, ainda resta estabelecer uma conexão mais consistente entre classificadores gerados off-board e sua utilização em sistemas embarcados, dada a pouca variedade de modelos de classificação empregados e a pouca disponibilidade de ferramentas que realizem adequadamente essa interface. Acredita-se que, dispondo de mais opções de classificadores para realização de testes, o usuário terá mais suporte ao avaliá-los, compará-los e determinar aqueles mais apropriados para o propósito desejado.

Neste artigo, é apresentada uma ferramenta de software que permite que modelos de classificação treinados offboard com a popular ferramenta Waikato Environment for Knowledge Analysis (WEKA) (Hall et al., 2009) sejam utilizados em sistemas embarcados de baixo custo. $\mathrm{O}$ foco da nossa ferramenta é gerar implementações de classificadores especificamente para esse tipo de sistema, com programação bare metal. Inclusive, as escolhas dos modelos de classificação suportados ratificam esse propósito, dado que são modelos simples, como regressão logística e árvore de decisão (J48), ou que já foram empregados com sucesso em aplicações embarcadas, como Multilayer Perceptron (MLP) e Support Vector Machine (SVM) (Shi et al., 2009; Rúa et al., 2012; Sampaio et al., 2017; Farooq et al., 2010; Tocchetto et al., 2014). A intenção é que nossa ferramenta seja genérica, suportando diferentes plataformas embarcadas, e que permita melhorar o desempenho dos classificadores através do uso de representação de pontofixo em vez de ponto flutuante. Além disso, propomos uma análise comparativa de desempenho dos classificadores a fim de dar suporte ao usuário na escolha do mais adequado para sua aplicação.

Este artigo está organizado da seguinte maneira: na Seção 2 é apresentado um apanhado geral das principais ferramentas utilizadas na área de AM para a geração de modelos de classificação; na Seção 3 são descritos trabalhos relacionados; na Seção 4 é apresentada a ferramenta proposta neste trabalho; na Seção 5 é apresentada a configuração experimental considerada para a avaliação da ferramenta proposta; na Seção 6, os resultados são apresentados e discutidos; por fim, na Seção 7 são apresentadas as conclusões e direcionamentos de trabalhos futuros.

\section{GERAÇÃO DE MODELOS DE CLASSIFICAÇÃO}

Determinadas ferramentas são populares e importantes para a comunidade de AM por disponibilizarem imple- mentações de diferentes algoritmos para as tarefas de classificação, agrupamento, regressão e outras. Além disso, permitem diversas funcionalidades aos usuários, como: salvar um modelo de AM treinado; aplicar técnicas de préprocessamento de dados; utilizar técnicas de seleção de atributos; empregar técnicas de avaliação de resultados; ajustar os parâmetros dos algoritmos; entre outras tarefas. Em vista disso, a ideia de utilizar classificadores produzidos por essas ferramentas é uma maneira de simplificar, ainda mais, o uso destes algoritmos em sistemas embarcados.

Dentre todas essas ferramentas, destacam-se algumas das mais populares que são capazes de desempenhar a maioria das funcionalidades citadas: WEKA (Hall et al., 2009), scikit-learn (Pedregosa et al., 2011), RapidMiner (Hofmann and Klinkenberg, 2013), KNIME (Berthold et al., 2007) e MOA (Bifet et al., 2010). Embora todas essas ferramentas apresentem características suficientemente interessantes para serem exploradas neste trabalho, foi escolhido a princípio, fornecer suporte apenas para modelos gerados pelo $W E K A$, uma vez que esta ferramenta disponibiliza implementações para os principais algoritmos de classificação e permite acesso aos códigos-fonte de tais algoritmos, facilitando a sua utilização. Em trabalhos futuros, as demais ferramentas serão suportadas em nossa proposta.

\section{TRABALHOS RELACIONADOS}

Nesta seção são descritos trabalhos com objetivos similares ao proposto neste artigo. Especificamente, programas responsáveis por converter modelos de classificação produzidos por ferramentas de AM em códigos-fonte que podem ser executados em microcontroladores.

Sklearn-porter ${ }^{1}$ é uma ferramenta que permite que alguns modelos treinados com scikit-learn sejam convertidos em implementações de classificadores (ou regressores) em diferentes linguagens de programação: Java, JavaScript, C, Go, PHP e Ruby. Por exemplo, há suporte para classificadores treinados pelos algoritmos SVM, Árvore de Decisão, Random Forest, Naive Bayes, K-Nearest Neighbors e MLP. Como a ferramenta é implementada na linguagem de programação Python, os modelos produzidos pela scikitlearn são passados como entrada em forma de objeto, desta mesma linguagem, que contém o classificador treinado. Após a execução, é gerado um arquivo de saída, na linguagem escolhida, contendo os dados do modelo de classificação com a implementação da função usada para classificar uma instância. Entretanto, é preciso observar que a ferramenta não permite adaptações no código e produz implementações em linguagens que não são populares para programar microcontroladores, sugerindo que esse não é o foco da ferramenta.

De maneira similar, a ferramenta weka-porter ${ }^{2}$ converte apenas modelos treinados no WEKA com o algoritmo $J 48$. Esta ferramenta deve receber como entrada um arquivo de texto que contém uma representação compacta de árvore de decisão, gerada pelo $W E K A$ no final da etapa de treinamento desse algoritmo. Ao final, é possível produzir classificadores implementados nas linguagens $C$, Java e JavaScript em formato similar aos produzidos pela ferramenta

\footnotetext{
1 https://github.com/nok/sklearn-porter

2 https://github.com/nok/weka-porter
} 
sklearn-porter. Novamente, a ferramenta gera código em linguagens que não são populares para microcontroladores e suporta apenas um modelo de classificação.

A ferramenta emlearn ${ }^{3}$ produz classificadores em $C$ a partir de modelos treinados com scikit-learn ou Keras. Essa ferramenta possui suporte para os algoritmos árvore de decisão, Naive Bayes, MLP e Random Forest. Além disso, os classificadores produzidos contêm características interessantes para plataformas embarcadas. Por exemplo, eles não utilizam alocação dinâmica de memória, possuem suporte para representação de ponto-fixo e não fazem uso de bibliotecas como stdlib. Apesar disso, há pouca diversidade de modelos suportados, não contendo classificadores como SVM, popular em aplicações embarcadas. E, atualmente, apenas o classificador Naive Bayes conta com suporte para operações em ponto-fixo.

Existem também ferramentas como $m S V M^{4}$ e $u L I B S V M^{5}$, desenvolvidas a partir de modificações realizadas na biblioteca libSVM (Chang and Lin, 2011), que disponibiliza implementações para o algoritmo SVM, e permitem produzir classificadores em linguagem $C++$ para executar em sistemas embarcados. Contudo, essas ferramentas são limitadas ao suporte de apenas um modelo de classificação.

Também pode-se citar outros exemplos de ferramentas que produzem classificadores em $C++$ a partir de modelos de árvore de decisão: $J_{48 t o C P P}{ }^{6}$ e $C 4.5$ decision tree $c p p^{7}$, capazes de converter modelos treinados pelo algoritmo $J_{48}$ do $W E K A$; e DecisionTreeToCpp ${ }^{8}$, capaz de converter modelos árvores de decisão do scikit-learn. Ou mesmo vale mencionar a ferramenta MATLAB Coder que permite gerar códigos em $\mathrm{C}$ e $\mathrm{C}++$ contendo modelos de AM para executar em diversas plataformas. Em todos esses casos, porém, não há diversidade de classificadores ou plataformas suportadas, ou são ferramentas para uso comercial com custo associado ao seu uso.

\section{FERRAMENTA PROPOSTA}

Neste artigo é proposta uma nova ferramenta capaz de converter automaticamente modelos gerados off-board em códigos que possam ser executados em sistemas embarcados. Especificamente, a ferramenta recebe como entrada um arquivo que contém um modelo de classificação serializado produzido pelo WEKA a partir de um conjunto de dados com atributos descritivos numéricos. Os valores das variáveis contidas no modelo são recuperados com o uso de ferramentas capazes de desserializar o arquivo. Então, é gerado um arquivo em linguagem de programação $C++$ contendo essas variáveis, seus valores de inicialização e implementações de funções que os utilizem para a classificação de uma instância. A ideia é que este arquivo de saída contenha funções que sejam utilizadas apenas com o propósito de classificação, sendo parte de um programa mais complexo que será executado em uma plataforma embarcada e que poderá conter outras funcionalidades de acordo com a aplicação.

\footnotetext{
3 https://github.com/emlearn/emlearn

4 https://github.com/chenguangshen/mSVM

5 https://github.com/PJayChen/uLIBSVM

6 https://github.com/mru00/J48toCPP

7 https://github.com/hatc/C4.5-decision-tree-cpp

8 https://github.com/papkov/DecisionTreeToCpp
}

A Figura 1 ilustra o processo de funcionamento da ferramenta. Vale observar que, após gerar o classificador, é possível que o usuário tenha que refazer o processo, no caso em que o classificador não atenda aos requisitos de tempo ou memória da aplicação, por exemplo. Assim, haveria uma retroalimentação no processo que não é ilustrada na figura, uma vez que depende de uma análise técnica ou experimental do usuário sobre o classificador.

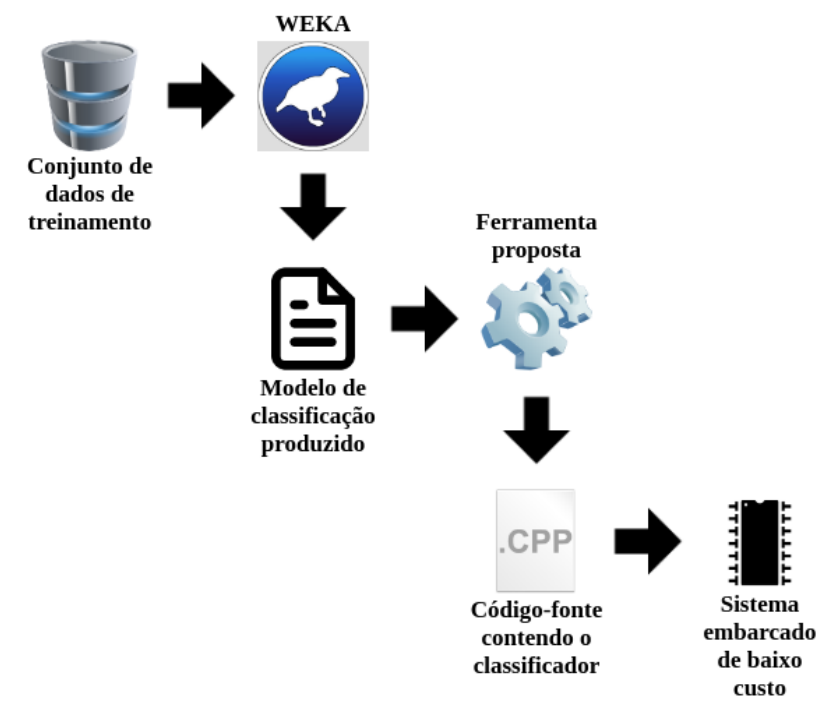

Figura 1. Processo de funcionamento da ferramenta.

O principal diferencial da ferramenta proposta é o foco na geração de modelos que possam ser executados em sistemas embarcados de baixo custo, com programação bare metal. Assim, as escolhas dos algoritmos suportados também consideram este objetivo, sendo modelos simples e que demandam pouco tempo de processamento.

\subsection{Serialização e recuperação de modelos}

Após treinar um modelo de classificação com a ferramenta $W E K A$, é possível salvá-lo para uso posterior. Para isso, realiza-se a serialização do objeto que contém o classificador em um arquivo, utilizando as classes ObjectOutputStream e FileOutputStream disponíveis em Java. Futuramente, esse objeto pode ser recuperado do arquivo que o contém por meio das classes ObjectInputStream e FileInputStream para ser utilizado em um código em Java.

Como as classes dos classificadores possuem a maioria de suas variáveis privadas, uma alternativa encontrada para ter acesso ao conteúdo das variáveis do modelo foi utilizar a biblioteca javaobj ${ }^{9}$ - disponível para a linguagem Python. Esta biblioteca permite recuperar um objeto do Java serializado em um arquivo e produzir uma estrutura em Python que contém as variáveis e dados contidos naquele. Assim, é possível ter acesso a todos os valores das variáveis do classificador treinado e selecionar o que é relevante para a etapa de classificação.

\subsection{Conceitos técnicos da implementação}

A versão implementada da ferramenta proposta provê suporte para modelos treinados com a ferramenta $W E K A$

\footnotetext{
9 https://pypi.org/project/javaobj-py3/
} 
que representam diferentes paradigmas de aprendizado. São eles: rede neural MLP, regressão logística, SVM (com kernel linear) e árvore de decisão (J48). As variáveis e estruturas do modelo treinado no $W E K A$ são recuperadas da maneira descrita anteriormente. Em seguida, a ferramenta produz um arquivo em linguagem de programação $C++$ contendo as variáveis e estruturas inicializadas, junto com implementações das funções necessárias para classificação - baseadas nas implementações do WEKA.

Ainda, a ferramenta dispõe de uma adaptação em que a implementação do classificador utiliza a representação de ponto-fixo Q21.10 para substituir a de ponto flutuante. Para tal, uma biblioteca de operações de pontofixo foi implementada com base em outras já existentes: fixedptc $^{10}$, libfixmath $^{11}$ e $A V R f i x^{12}$. O uso de representação de ponto-fixo visa melhorar o tempo de execução dos classificadores principalmente em microcontroladores que não possuem unidade de ponto flutuante, uma vez que essas operações podem ser bastante custosas nesse caso.

\section{CONFIGURAÇÃO EXPERIMENTAL}

São apresentados a seguir os conjuntos de dados e plataformas embarcadas consideradas na avaliação experimental.

\subsection{Conjuntos de dados}

A avaliação experimental foi conduzida com seis conjuntos de dados provenientes de aplicações reais que envolvem o uso de sensores. A escolha destes problemas se deve ao fato de que, em geral, aplicações de sensores fazem uso de sistemas embarcados para a realização da tarefa de classificação por meio de dispositivos de baixo custo. Uma descrição destes conjuntos de dados é apresentada a seguir:

Aedes aegypti-sex (dos Reis et al., 2018). Este conjunto é composto por dados obtidos por um sensor óptico capaz de medir a frequência de batida de asas de insetos alados. Basicamente, a tarefa é identificar em tempo real o sexo de insetos da espécie Aedes aegypti;

Asfault-streets (Souza et al., 2018). Este conjunto considera um problema de avaliação em tempo real da qualidade do asfalto de ruas utilizando um sensor de acelerômetro no interior de um automóvel. O asfalto pode ser classificado em: bom, regular, ruim, péssimo e obstáculo.

Asfault-roads (Souza et al., 2018). Este conjunto de dados é uma variação do problema anterior em que considerase a avaliação de estradas. Neste caso, a categoria "péssimo" não está presente no conjunto de dados.

GasSensorArray (Vergara et al., 2012). Este conjunto de dados consiste de medições ao longo do tempo de 16 sensores químicos com o objetivo de identificar os seguintes gases em uma plataforma de gás: amônia, acetaldeído, acetona, etileno, etanol e tolueno.

PenDigits (Alimoglu and Alpaydin, 1996). Este conjunto de dados envolve um problema de classificação de dígitos manuscritos (0 a 9 ) a partir das coordenadas $x$ e $y$ de uma caneta em uma tela digital.

\footnotetext{
${ }_{10}$ https://sourceforge.net/projects/fixedptc/

11 https://code.google.com/archive/p/libfixmath/

12 https ://sourceforge.net/projects/avrfix/
}

HAR (Reyes-Oritz et al., 2012). Este conjunto de dados considera o problema de classificação de atividades humanas a partir da análise de dados de acelerômetro. São consideradas as seguintes atividades: caminhando, subindo escadas, descendo escadas, sentado, em pé e deitado.

Os conjuntos de dados avaliados possuem variabilidades entre si em termos de quantidade de exemplos, número de atributos descritivos e quantidade de classes. As principais características são apresentadas na Tabela 1 .

Tabela 1. Características dos conjuntos de dados.

\begin{tabular}{lccc}
\hline Nome & Atributos & Classes & Exemplos \\
\hline Aedes aegypti-sex & 42 & 2 & 42.000 \\
Asfault-streets & 64 & 5 & 3.878 \\
Asfault-roads & 64 & 4 & 4.688 \\
GasSensorArray & 128 & 6 & 13.910 \\
PenDigits & 8 & 10 & 10.992 \\
HAR & 561 & 6 & 10.299 \\
\hline
\end{tabular}

Com o objetivo de padronizar a configuração experimental e a análise de resultados, foi utilizado o método de validação holdout 70/30. Assim, cada um dos conjuntos de dados foi dividido em dois subconjuntos estratificados e mutuamente exclusivos, em que $70 \%$ dos dados foi utilizado para a construção do modelo de classificação e os $30 \%$ restantes dos dados foram utilizados na etapa de teste. Em relação aos parâmetros dos algoritmos, considerou-se os valores padrões definidos no $W E K A$.

\subsection{Plataformas embarcadas}

Considerando a vasta quantidade de plataformas embarcadas de baixo custo existentes e que aceitam programação bare metal, escolheu-se avaliar os classificadores em um modelo simples e popular (Arduino Mega 2560), outro com capacidade de processamento intermediária (Teensy 3.2 ) e outro que dispõe de unidade de ponto flutuante (Teensy 3.6). Os modelos da plataforma Teensy possuem microcontroladores de 32 bits que utilizam núcleos da família ARM Cortex-M4 e o modelo Arduino Mega 2560 possui um microcrontrolador ATmega2560 de 8 bits e de baixa potência.

A Tabela 2 apresenta algumas especificações das plataformas embarcadas selecionados, expondo principalmente características de seus microcontroladores relacionadas a disponibilidade de memória (SRAM e flash) e capacidade de processamento (frequência de clock), que são úteis para a comparação do desempenho dos classificadores.

Tabela 2. Características das plataformas embarcadas.

\begin{tabular}{lccc}
\hline Plataforma & clock & SRAM & flash \\
\hline Arduino Mega 2560 & $16 \mathrm{MHz}$ & $8 \mathrm{kB}$ & $256 \mathrm{kB}$ \\
Teensy 3.2 & $72 \mathrm{MHz}$ & $64 \mathrm{kB}$ & $256 \mathrm{kB}$ \\
Teensy 3.6 & $180 \mathrm{MHz}$ & $256 \mathrm{kB}$ & $1024 \mathrm{kB}$ \\
\hline
\end{tabular}

\section{ANÁLISE DE RESULTADOS}

Para validar os classificadores produzidos pela ferramenta proposta, foram utilizados os conjuntos de dados descritos com os quais modelos de classificação foram treinados na 
ferramenta $W E K A$. Após treinados, esses modelos foram utilizados por nossa ferramenta para produzir arquivos em linguagem de programação $C++$ contendo os classificadores que foram executados nas plataformas embarcadas. A programação ocorreu com o uso da ferramenta PlatformIO ${ }^{13}$ - que também permite programar outras plataformas na mesma linguagem.

As instâncias do conjunto de teste foram armazenadas em um cartão de memória microSD para serem usadas pelos microcontroladores. Mesmo assim, não foi possível realizar alguns testes, pois o consumo de memória do classificador foi superior à capacidade de armazenamento do microcontrolador.

Em todas execuções realizadas dos classificadores (com representação de ponto flutuante) nas plataformas escolhidas, foram obtidos os mesmos valores de acurácia no teste em comparação aos obtidos na ferramenta $W E K A$, exceto no caso do modelo MLP para o conjunto Asfaultroads em que a acurácia obtida no WEKA foi de $89,19 \%$. As demais acurácias são apresentadas na Tabela 3.

Tabela 3. Acurácia (\%) para os classificadores com representação de ponto flutuante.

\begin{tabular}{lcccc}
\hline \multicolumn{1}{c}{$\begin{array}{c}\text { Conjunto } \\
\text { de dados }\end{array}$} & SVM & $\mathbf{J 4 8}$ & MLP & $\begin{array}{c}\text { Regressão } \\
\text { logística }\end{array}$ \\
\hline Aedes aegypti-sex & 98,39 & $\mathbf{9 9 , 0 0}$ & 98,67 & 97,71 \\
Asfault-streets & $\mathbf{9 1 , 7 5}$ & 84,28 & 90,29 & 89,00 \\
Asfault-roads & $\mathbf{9 1 , 9 6}$ & 88,48 & 89,26 & 91,61 \\
GasSensorArray & 97,13 & 97,41 & 92,84 & $\mathbf{9 8 , 9 7}$ \\
PenDigits & 80,67 & $\mathbf{8 4 , 7 1}$ & 79,37 & 73,00 \\
HAR & $\mathbf{9 8 , 4 1}$ & 94,34 & 81,84 & 97,57 \\
\hline
\end{tabular}

A Tabela 4 apresenta as acurácias obtidas no teste provenientes das execuções, nas plataformas, dos classificadores com representação de ponto-fixo Q21.10. A maior diferença dos valores de acurácia obtidos nas execuções do mesmo classificador com representação de ponto flutuante e de ponto-fixo foi de 1,07\% (MLP e Asfault-roads).

Tabela 4. Acurácia (\%) para os classificadores com representação de ponto-fixo.

\begin{tabular}{lcccc}
\hline $\begin{array}{c}\text { Conjunto } \\
\text { de dados }\end{array}$ & SVM & $\mathbf{J 4 8}$ & MLP & $\begin{array}{c}\text { Regressão } \\
\text { logística }\end{array}$ \\
\hline Aedes aegypti-sex & 98,40 & $\mathbf{9 8 , 9 7}$ & 98,65 & 97,65 \\
Asfault-streets & $\mathbf{9 1 , 9 2}$ & 84,54 & 90,46 & 87,97 \\
Asfault-roads & $\mathbf{9 2 , 3 2}$ & 88,41 & 90,33 & 91,54 \\
GasSensorArray & 97,13 & 97,41 & 92,86 & $\mathbf{9 8 , 3 5}$ \\
PenDigits & 80,67 & $\mathbf{8 4 , 7 1}$ & 79,46 & 73,21 \\
HAR & $\mathbf{9 7 , 9 9}$ & 94,01 & - & 97,54 \\
\hline
\end{tabular}

Uma comparação de tempo de classificação foi realizada nas plataformas embarcadas, medindo-se, nas instâncias do conjunto de teste, tempo médio e tempo máximo de classificação. Para todos os gráficos a seguir, são apresentados o tempo máximo (em $\mu s$ ) e o tempo médio (em $\mu s)$ para classificar uma instância, obtidos a partir de 10 execuções do classificador em cada instância do conjunto de teste. Essas medidas são referentes apenas à etapa de classificação e foram realizadas por meio da função micros $^{14}$. Além disso, os gráficos apresentam os valores de

\footnotetext{
13 https://platformio.org/

14 https ://www .arduino.cc/reference/en/language/functions/ time/micros/
}

tempo em escala logarítmica para facilitar a visualização. Cada barra contém as informações de tempo médio (em vermelho) e tempo máximo (em azul) de classificação, sendo que em muitos casos não é possível visualizar a porção azul por conta da pouca diferença entre esses valores. As barras que representam valores de tempo de execução com representações de ponto flutuante (FLT) e ponto-fixo (FXP), para a mesma placa e classificador, estão mais próximas a fim de favorecer a comparação desses.

As Figuras 2, 3, 4, 5, 6 e 7 apresentam os gráficos em escala logarítmica dos tempos obtidos para os conjuntos de dados Aedes-sex, Asfault-streets, Asfault-roads, GasSensorArray, PenDigits e HAR, respectivamente. Os casos ausentes são aqueles para os quais não foi possível realizar os testes por limitação de memória do microcontrolador.

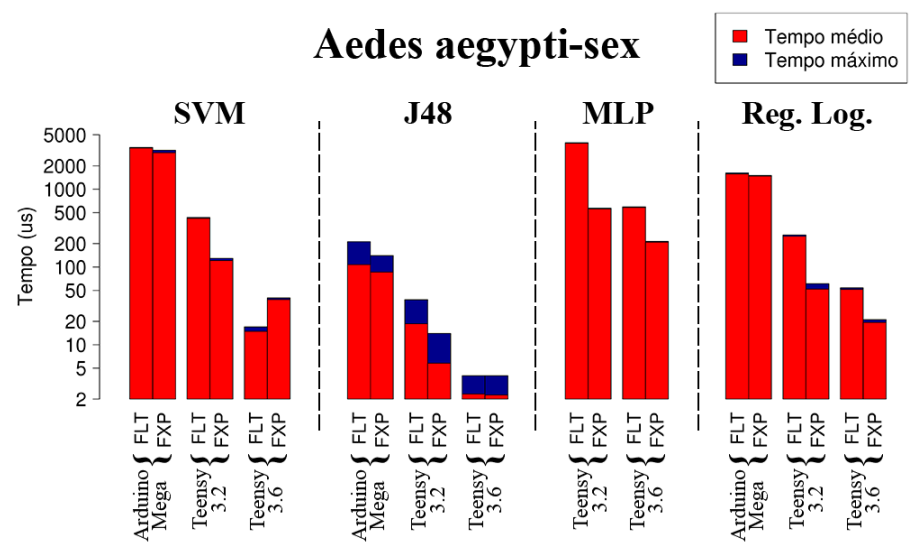

Figura 2. Desempenho dos classificadores no teste com o conjunto Aedes aegypti-sex.

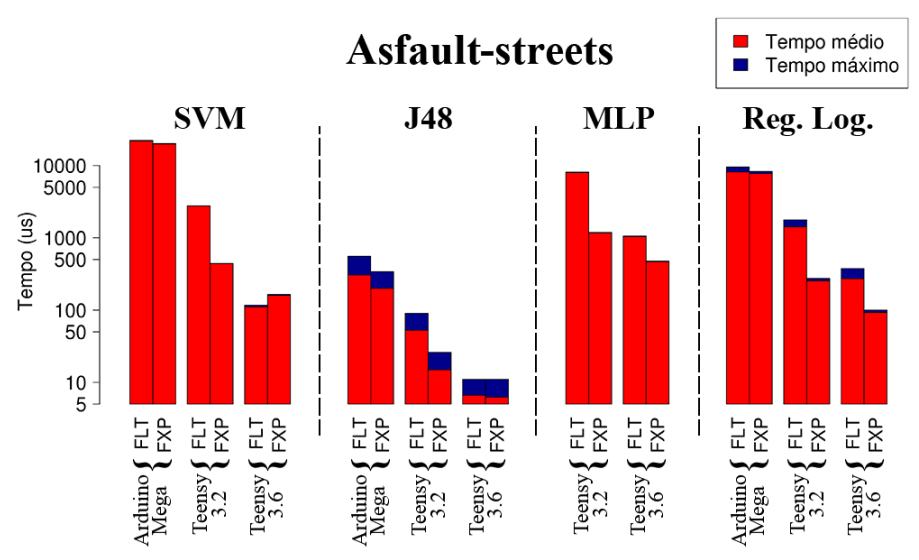

Figura 3. Desempenho dos classificadores no teste com o conjunto Asfault-streets.

Para cada conjunto de dados, o maior e menor valor de tempo máximo de classificação por instância são destacados na Tabela 5 junto com a combinação de classificador, representação e plataforma utilizada para obtê-los.

Considerando a comparação de tempo máximo de classificação entre as execuções das representações avaliadas para o mesmo classificador, temos que, ao utilizar representação de ponto-fixo, houve uma redução de 92,90\% do tempo de classificação no melhor caso (PenDigits, Reg. Log., Teensy 3.2), passando de $5420 \mu \mathrm{s}$ (FLT) para $385 \mu \mathrm{s}$ (FXP). No pior caso (Aedes aegypti-sex, SVM, Teensy 3.6), o 


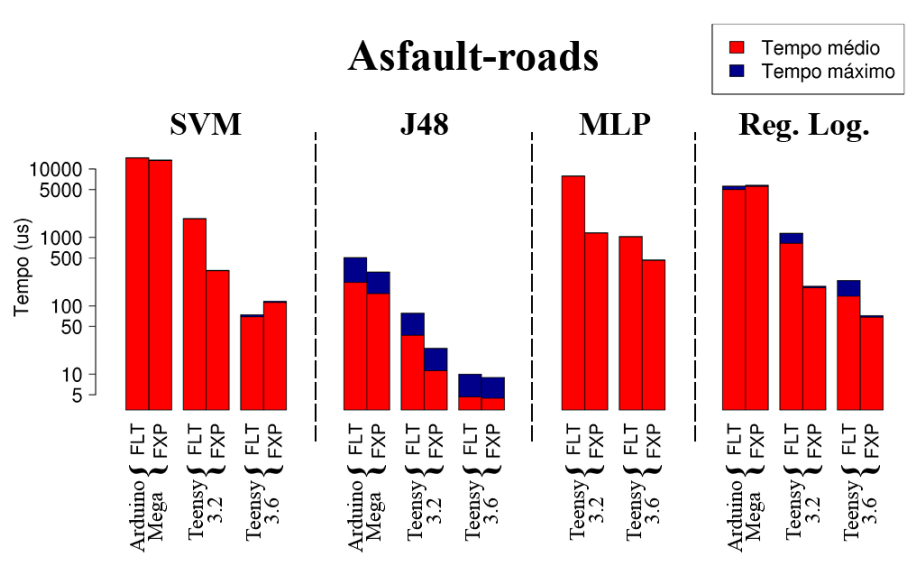

Figura 4. Desempenho dos classificadores no teste com o conjunto Asfault-roads.

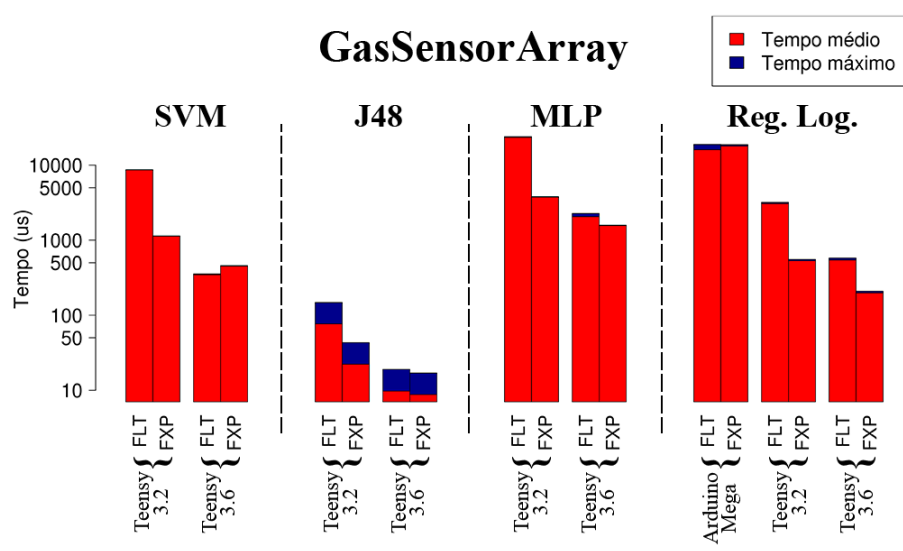

Figura 5. Desempenho dos classificadores no teste com o conjunto GasSensorArray.

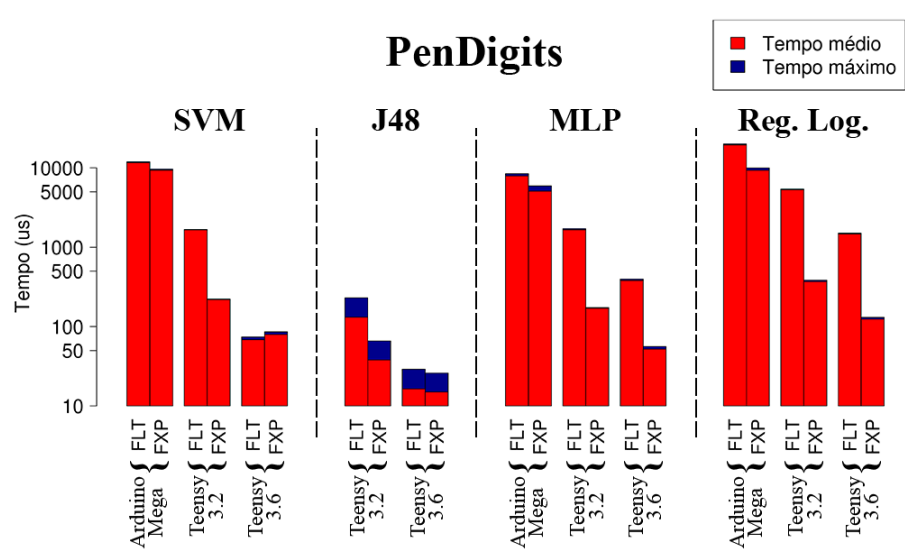

Figura 6. Desempenho dos classificadores no teste com o conjunto PenDigits.

tempo de classificação aumentou $135,29 \%$, passando de $17 \mu s$ (FLT) para 40 $\mu s$ (FXP).

Para fins de reprodução dos resultados apresentados neste artigo e uso de nossa ferramenta, disponibilizamos em um repositório online ${ }^{15}$ a implementação da ferramenta, os modelos do WEKA serializados, os classificadores produzidos e detalhes dos resultados obtidos.

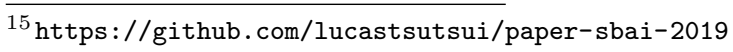

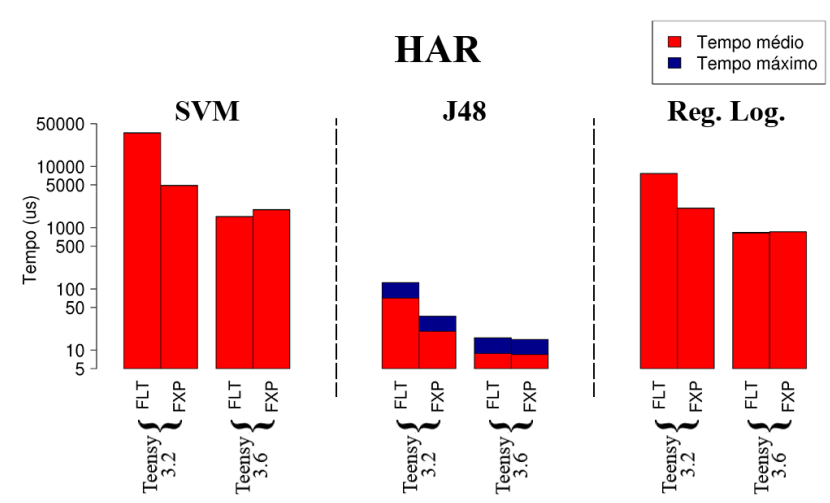

Figura 7. Desempenho dos classificadores no teste com o conjunto HAR.

Tabela 5. Detalhes do tempo de execução dos classificadores.

\begin{tabular}{|c|c|c|}
\hline $\begin{array}{l}\text { Conjunto } \\
\text { de dados }\end{array}$ & $\begin{array}{l}\text { Maior tempo } \\
\text { máximo } \\
\text { (classificador, } \\
\text { representação, } \\
\text { plataforma) }\end{array}$ & $\begin{array}{l}\text { Menor tempo } \\
\text { máximo } \\
\text { (classificador, } \\
\text { representação, } \\
\text { plataforma) }\end{array}$ \\
\hline Aedes aegypti-sex & $\begin{array}{c}\text { 3962 } \mu s \\
(\mathrm{MLP}, \mathrm{FLT}, \\
\text { Teensy 3.2) }\end{array}$ & $\begin{array}{c}4 \mu s \\
(\mathrm{~J} 48, \text { FLT e FXP } \\
\text { Teensy 3.6) }\end{array}$ \\
\hline Asfault-streets & 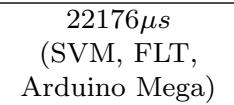 & $\begin{array}{c}11 \mu s \\
(\mathrm{~J} 48, \text { FLT e FXP } \\
\text { Teensy 3.6) }\end{array}$ \\
\hline Asfault-roads & $\begin{array}{c}14584 \mu s \\
\text { (SVM, FLT, } \\
\text { Arduino Mega) }\end{array}$ & $\begin{array}{c}9 \mu s \\
(\mathrm{~J} 48, \mathrm{FXP}, \\
\text { Teensy 3.6) }\end{array}$ \\
\hline GasSensorArray & $\begin{array}{c}24034 \mu s \\
\text { (MLP, FLT, } \\
\text { Teensy 3.2) }\end{array}$ & $\begin{array}{c}17 \mu s \\
(\mathrm{~J} 48, \mathrm{FXP}, \\
\text { Teensy 3.6) }\end{array}$ \\
\hline PenDigits & $\begin{array}{c}20072 \mu s \\
\text { (Reg. Log., FLT, } \\
\text { Arduino Mega) }\end{array}$ & $\begin{array}{c}26 \mu s \\
(\mathrm{~J} 48, \mathrm{FXP}, \\
\text { Teensy 3.6) }\end{array}$ \\
\hline HAR & $\begin{array}{c}35543 \mu s \\
(\mathrm{SVM}, \mathrm{FLT}, \\
\text { Teensy 3.2) }\end{array}$ & $\begin{array}{c}15 \mu s \\
(\mathrm{~J} 48, \mathrm{FXP}, \\
\text { Teensy 3.6) }\end{array}$ \\
\hline
\end{tabular}

\section{CONCLUSÕES}

Neste trabalho, apresentamos uma ferramenta que produz classificadores para sistemas embarcados de baixo custo a partir de modelos treinados off-board com a ferramenta $W E K A$. Para validar os classificadores gerados por nossa ferramenta, foram conduzidos experimentos com diferentes conjuntos de dados em modelos de plataformas embarcadas com características distintas. No aspecto geral, os principais resultados foram que os valores de acurácia obtidos no teste com o $W E K A$ se mantiveram (na maioria dos casos) em comparação aos obtidos nas plataformas embarcadas e o uso da representação de ponto-fixo se mostrou uma adaptação interessante, pois causou pouca alteração no valor de acurácia dos modelos e, no geral, melhorou o tempo máximo de classificação por instância, produzindo uma redução de tempo de até 92,90\% ao comparar com a mesma versão do classificador operando com ponto flutuante.

É importante destacar que, apesar dos modelos J48 alcançarem os melhores valores de tempo de classificação, não foi possível executá-los na plataforma Arduino Mega 
para metade dos conjuntos de dados, por excederem a quantidade disponível de memória do microcontrolador. Isso também ocorreu com os modelos MLP na maioria dos testes. Portanto, é necessário ter mais precaução ao decidir empregar esses classificadores, principalmente em aplicações em que a disponibilidade de memória é bastante limitada.

Por outro lado, em metade dos conjuntos de dados, os modelos SVM obtiveram os melhores valores de acurácia nos testes e, em contraste, maiores valores de tempo máximo de classificação. Já os classificadores de regressão logística tiveram um desempenho intermediário considerando o tempo de classificação e acurácia nos testes, porém conseguiram executar na plataforma Arduino Mega para a maior quantidade de conjuntos de dados.

Como trabalhos futuros, pretendemos ampliar o suporte de nossa ferramenta para modelos de classificação treinados com scikit-learn, bem como propor e avaliar outras adaptações para os classificadores que poderão melhorar o desempenho, como armazenar os dados na memória flash do microcontrolador e utilizar a representação Logarithmic Number System (Stouraitis, 1986). Além disso, resta realizar uma análise do consumo de memória dos classificadores produzidos pela ferramenta, a fim de que se desenvolva uma comparação de desempenho mais sustentada e aprofundada. Por fim, espera-se avaliar os classificadores em uma aplicação prática para reconhecimento automático de diferentes espécies de insetos alados em tempo real (Souza et al., 2013; Qi et al., 2015; Silva et al., 2015).

\section{AGRADECIMENTOS}

Os autores gostariam de agradecer a Fundação de Amparo à Pesquisa do Estado de São Paulo (FAPESP) (\#16/04986-6 e \#18/05859-3), CNPq (306631/2016-4 e $166919 / 2017-9$ ) e USAID (AID-OAA-F-16-00072) pelo apoio financeiro.

\section{REFERÊNCIAS}

Alimoglu, F. and Alpaydin, E. (1996). Methods of combining multiple classifiers based on different representations for pen-based handwritten digit recognition. In Proceedings of the Turkish Artificial Intelligence and Artificial Neural Networks Symposium.

Berthold, M.R., Cebron, N., Dill, F., Gabriel, T.R., otter, T.K., Meinl, T., Ohl, P., Sieb, C., Thiel, K., and Wiswedel, B. (2007). KNIME: The Konstanz Information Miner. In Studies in Classification, Data Analysis, and Knowledge Organization.

Bifet, A., Holmes, G., Kirkby, R., and Pfahringer, B. (2010). MOA: massive online analysis. Journal of Machine Learning Research, 11, 1601-1604.

Chang, C.C. and Lin, C.J. (2011). LIBSVM: A library for support vector machines. ACM Transactions on Intelligent Systems and Technology, 2, 27:1-27:27.

dos Reis, D.M., Maletzke, A.G., and Batista, G.E.A.P.A. (2018). Unsupervised context switch for classification tasks on data streams with recurrent concepts. In $A C M$ $S A C, 518-524$. ACM.

Farooq, U., Amar, M., ul Haq, E., Asad, M.U., and Atiq, H.M. (2010). Microcontroller based neural network controlled low cost autonomous vehicle. In ICMLC, 96100.

Hall, M., Frank, E., Holmes, G., Pfahringer, B., Reutemann, P., and Witten, I.H. (2009). The weka data mining software: an update. ACM SIGKDD explorations newsletter, 11(1), 10-18.

Hofmann, M. and Klinkenberg, R. (2013). RapidMiner: Data mining use cases and business analytics applications. CRC Press.

Pedregosa, F., Varoquaux, G., Gramfort, A., Michel, V., Thirion, B., Grisel, O., Blondel, M., Prettenhofer, P., Weiss, R., Dubourg, V., Vanderplas, J., Passos, A., Cournapeau, D., Brucher, M., Perrot, M., and Duchesnay, E. (2011). Scikit-learn: Machine learning in Python. Journal of Machine Learning Research, 12, 2825-2830.

Qi, Y., Cinar, G.T., Souza, V.M.A., Batista, G.E.A.P.A., Wang, Y., and Principe, J.C. (2015). Effective insect recognition using a stacked autoencoder with maximum correntropy criterion. In IJCNN, 1-7. IEEE.

Reyes-Oritz, J., Anguita, D., Ghio, A., Oneto, L., and Parra, X. (2012). Human activity recognition using smartphones data set. UCI Machine Learning Repository; University of California, Irvine, School of Information and Computer Sciences: Irvine, CA, USA.

Rúa, S., Zuluaga, S.A., Redondo, A., Orozco-Duque, A., Restrepo, J.V., and Bustamante, J. (2012). Machine learning algorithms for real time arrhythmias detection in portable cardiac devices: microcontroller implementation and comparative analysis. In STSIVA, 50-55.

Sampaio, F., da Silva, L.C., Reboucas Filho, P.P., and da Silva, E.T. (2017). Reducing computational costs of an embedded classifier to determine leather quality. In $S B E S C, 211-216$.

Shi, G., Chan, C.S., Li, W.J., Leung, K.S., Zou, Y., and Jin, Y. (2009). Mobile human airbag system for fall protection using mems sensors and embedded svm classifier. IEEE Sensors Journal, 9(5), 495-503.

Silva, D.F., Souza, V.M.A., Ellis, D.P.W., Keogh, E.J., and Batista, G.E.A.P.A. (2015). Exploring low cost laser sensors to identify flying insect species. Journal of Intelligent \& Robotic Systems, 80(1), 313-330.

Souza, V.M.A., Silva, D.F., and Batista, G.E.A.P.A. (2013). Classification of data streams applied to insect recognition: Initial results. In Brazilian Conference on Intelligent Systems, 76-81. IEEE.

Souza, V.M.A., Giusti, R., and Batista, A.J.L. (2018). Asfault: A low-cost system to evaluate pavement conditions in real-time using smartphones and machine learning. Pervasive and Mobile Computing, 51, 121-137.

Stankovic, J.A. (1988). Misconceptions about real-time computing: A serious problem for next-generation systems. Computer, 21(10), 10-19.

Stouraitis, A.G. (1986). Logarithmic number system theory, analysis and design. Ph.D. thesis, University of Florida.

Tocchetto, M.A., Bazanella, A.S., Guimaraes, L., Fragoso, J., and Parraga, A. (2014). An embedded classifier of lung sounds based on the wavelet packet transform and ann. IFAC Proceedings Volumes, 47(3), 2975-2980.

Vergara, A., Vembu, S., Ayhan, T., Ryan, M.A., Homer, M.L., and Huerta, R. (2012). Chemical gas sensor drift compensation using classifier ensembles. Sensors and Actuators B: Chemical, 166, 320-329. 